\title{
Extracting features from laser scanning point cloud $^{a}$
}

\author{
Vladimir Badenko ${ }^{1 *}$, Alexander Fedotov ${ }^{1}$, and Dmitry Zotov ${ }^{1}$ \\ ${ }^{1}$ Peter the Great St. Petersburg Polytechnic University, High-performance hybrid geospatial data \\ systems Centre, 195251, Polytechnicheskaya, 29, Russian Federation
}

\begin{abstract}
Analyses of gaps in processing of raw laser scanning data and results of bridging the gaps discovered on the basis of usage of laser scanning data for historic building information modelling are presented. Some results of the development of a unified hybrid technology for the processing, storage, access and visualization of combined laser scanning and photography data about historical buildings are analyzed. The first result of the technology application to historical building of St. Petersburg Polytechnic University shows the robustness of the approaches proposed.
\end{abstract}

\section{Introduction}

The laser scanning is the most up-to-date and actively developing type of the remote sounding technologies. Presently, these 3D technologies are used all over the world in a wide spectrum of applications: roads management, urban modeling, departmental investigations and cultural heritage preservation [1]. The corresponding types of data acquisition systems are as follows: ground, mobile and airborne laser scanning [2]. The laser scanning is one of the most active methods of remote sounding. The principle of laser scanners operation, irrespective of the type and purpose thereof, is based on measuring distance from the laser pulse source to an object. In the course of object surveying the required distance will be determined with respect to time delay (pulse-type method) or to phase shift (phase method) by a signal reflected by the object surface [3]. The laser scanning result will be a cloud of points, which is generally coupled with photos [4]. Thus, a user can have the following information for every point of a cloud: three coordinates, reflected signal intensity and color (R, G, B), which precisely describe an object under investigation. A geometrical accuracy and density of points of such clouds have significantly increased recently [5].

The purpose of this article consists in the analysis of some deficiencies in processing the unprocessed laser scanning data and presentation of some results of rectifying deficiencies revealed on the basis of using the laser scanning data for historic building information modelling. The article presents the results of processing a unified hybrid technology for

\footnotetext{
${ }^{a}$ The research was supported by Ministry of Education and Science of Russia within the framework of the Federal Program "Research and Development in Priority Areas for the Development of the Russian the Science and Technology Complex for 2014-2020" (project ID RFMEFI58417X0025).

*Corresponding author: vbadenko@gmail.com
} 
processing, storage, access and visualization of combined data of laser scanning and photos of historic buildings.

\section{Materials and Methods}

An experience of architectural measurements of historic buildings with the use of laser scanning technologies has demonstrated that the measurement drawings of facades and interiors of buildings are being most efficiently built on the basis of raster ortho-images, which are obtained by means of projecting a cloud of laser points on the image plane. A similar approach is traditionally used in photographic survey [6]. The mobile laser scanning (MLS) is the most advanced and the least widespread technology in Russia. However, the number of MLS equipment in Russia has already exceeded the number of airborne laser scanners, while an amount of information per unit of time received by MLS scanner (up to 3-5 million points per second) several times exceeds the volume created during airborne scanning. There is a number of promising applications requiring collection of geospatial data with the use of MLS. In this case the processing of MLS data is developed insufficiently.

The analysis of the current state of investigations in this field has shown that there exists a great number of publications dedicated to the tasks of plotting digital models of buildings, detecting ground surface and reconstruction of road pavement, revealing and recognizing the road objects on the basis of MLS data $[5,7,8]$.

In spite of the available successes, one can distinguish the following algorithms demanding more profound studying:

- for hierarchic processing of a great amount of data, which provide for hybrid processing and compression of data without loss of accuracy,

- for integration of scanning data received from different sources,

- for seamless combination of real-time data processing during shaping orthogonal image based on a single scanning and on the series scans received from different sources, for providing reduction of "dead zones" area.

The offered method corresponds to a complex problem solution through establishing an algorithmic support of hybrid data processing system received from all types of laser scanning (mobile, ground and airborne), as well as photos. A solution offered by the authors corresponds not to a general 3D modeling of urban districts or, for instance, historic buildings and structures. An offered approach provides for a method of automatic and semiautomatic processing and visualization of data on the basis of forming cylindrical scans for point clouds. In order to solve the problems of historic building information modeling (HBIM) [9], a method of big data volumes processing has been developed on the basis of laser scanning data. The method provides for more precise HBIM particularization, including a combination of scanning data for every scale and level of providing information of studies received by different scanning methods.

The determination of factors and assessment of indicators for factors influencing the accuracy are not analyzed in this method. Special approaches to compressing data without losing accuracy and reduction of "dead zones" area in ortho-images are used. A task of defining geometry of buildings is solved by the existing methods with the use of integrated information received by different methods with the use of interpolation and extrapolation of acquired data. However, an experimental investigation of efficiency of different methods of surface modeling has been carried out for providing an acceptable level of accuracy to support the suggested method. 


\section{Results}

The investigation goal consists in the development of new algorithms of processing of cloud points (big data processing) received from the laser scanner. The creation of software for processing, visualization and vectorization of laser scanning data as well as data presentation via Internet, providing:

(1) increasing the efficiency and accuracy of processing laser scanning data (decoding and vectorization not less than $99.5 \%$ );

(2) provide the laser scanning customer (in the framework of engineering studies) with functionality of subsequent processing of scanning results on the basis of primary (unprocessed) information about the object (which is the most valuable information).

The investigations of offered technology have been tested in the course of creating 3D data for the buildings of Peter the Great St. Petersburg Polytechnic University (former Petersburg Polytechnic Institute). St. Petersburg Polytechnic Institute has been built by the example of Oxford and Cambridge as a complex with carefully designed and incorporated infrastructure. The construction of the main buildings of the Institute was performed according to a design of architectural engineer E. Virich, architectural engineer of the $1^{\text {st }}$ degree, Academician of architecture since 1908, an outstanding representative of rationalistic architecture, one of the pioneers in using reinforced-concrete structures in 1900-1905.

The Polytechnic Institute complex is interesting as a coherent architectural ensemble, one of the few that has been preserved in the relatively unchanged form. The buildings correspond to unique architectural buildings of the boundary of XIX - XX centuries. A complex of buildings and a park is included into a "List of historical and cultural heritage sites of federal significance" approved by the decree of the President of the Russian Federation No. 176 dated February 20, 1995 and is an especially valuable cultural heritage site of the peoples of the Russian Federation as the complex historical, cultural, architectural ensemble and structures as well as a monument to the natural landscape representing a material, intellectual and decorative value. One of the oldest structures in the University building is a water supply tower built in 1905 (Fig. 1a), three years after PolytechnicInstitute opening. It is the highest point among two- and four-storied institute buildings, and it stands in contrast to the other structures due to its intricate and polygonal shapes. The height of water supply tower is 46 meters. Before 1953 it was used as an engineering structure for providing operation of water pipelines system. The built supply water tower became one of the educational buildings in the territory of the Institute. In 1905 the Laboratory of hydraulics was installed in the additional building close to the water supply tower.

There exists a multiplicity of variants of building digital models of big objects, including photographic survey and 3D laser scanning. The complexity of investigation has demanded utilization of both methods. In order to build 3D models for HBIM of historical heritage, the methods of 3D laser-scanning and photographic-survey data processing. 


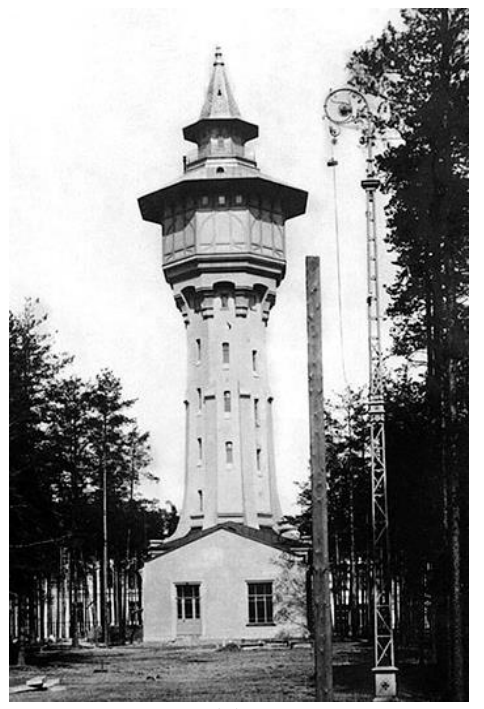

(a)

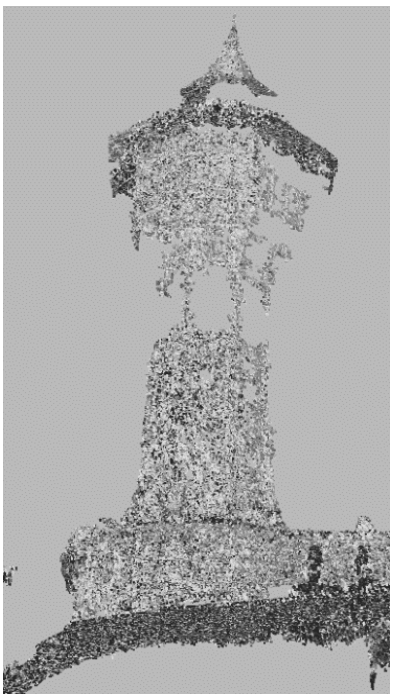

(b)

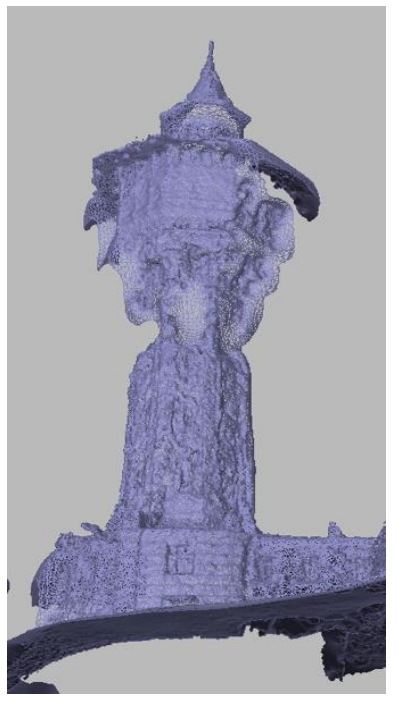

(c)

Fig. 1. (a) Water-Supply Tower in 1905; (b) Cloud of Points for Historic Building; (c) Result of Semiautomatic Triangulation.

A building of 3D model includes the following steps: selecting investigation object; collecting visual, technical and historical information; laser scanning and photographic survey for acquisition of a cloud of data points (Fig.1b); detection and extraction of segments of a dense cloud of points and a procedure of semiautomatic triangulation (Fig. 1b); obtaining external color models with the use of photos (Fig.2a); data export into applied architectural packages (Fig.2b, Fig.2c).

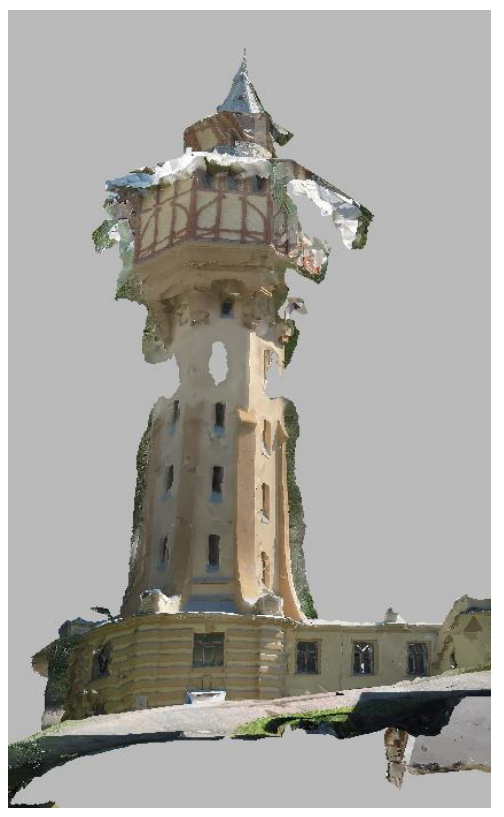

(a)

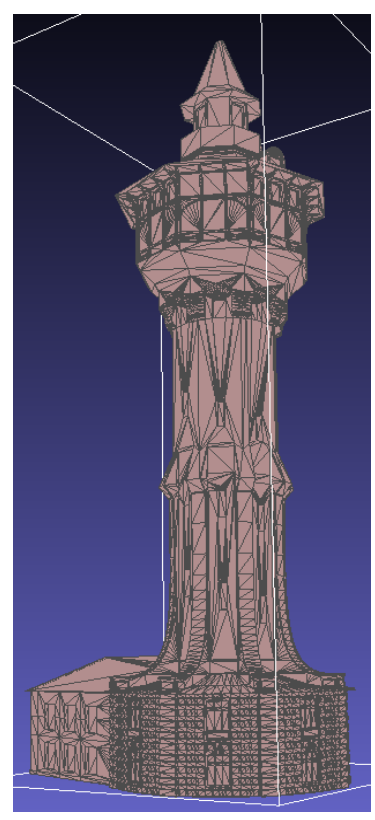

(b)

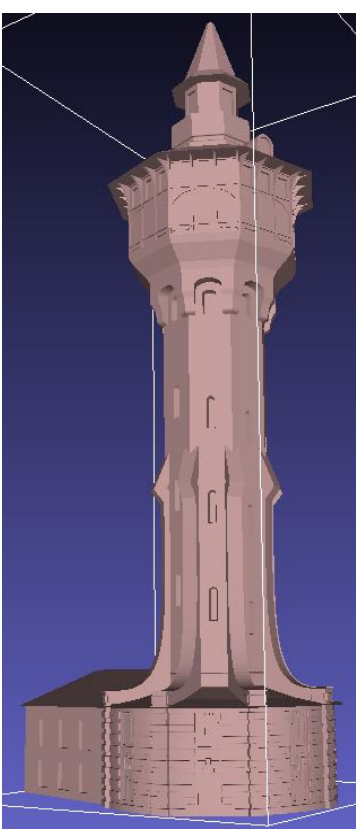

(c)

Fig. 2. (a) External Color Model; (b) 3D Grid for BIM; (c) Model of 3D BIM 
A dense cloud of points has been built (Fig.1a) on the basis of comparing images with the use of OpenCV library [10]. A dense cloud of points is generally understood as a cloud featuring density of at least 1 point per $1 \mathrm{~cm}^{2}$ in case of surveying big objects. In case of this object the cloud of points amounted to $32 \mathrm{KK}$ points. The processing of this number of points has no sense and is extremely expensive in terms of hardware resources. In order to do so, it is necessary to reduce a number of points. An optimal solution for big objects featuring intricate geometry is to reduce the number of points 10 times up to $3.2 \mathrm{KK}$

\section{Discussion}

The engineering study for the existing building is an activity, where the up-to-date high-tech laser scanners and equipment are used more and more frequently [11]. The corresponding methods are used for surveying different objects, beginning from historic buildings and ending with uninhabited territories for road construction $[4,12,13]$. The laser scanning systems acquired data more completely and effectively than the traditional survey methods do [14]. A future development of these systems provides for emergence of a compact, light and relatively inexpensive multipurpose of laser scanning, which shall be fast and simple for deployment and require minimal quantity of the existing equipment for operative support [1]. The known specific features of laser scanning data are as follows: high accuracy and density resulting in building big data volumes - spatial clouds of points [15]. A process of acquisition of the clouds of points for every type of laser scanning is well explored, and the corresponding software of acquisition of the cloud of points will be provided by a manufacturer [16]

An essential drawback of laser scanning technologies, which significantly restricts its use, is a permanent gap between quick development of technologies and capabilities of processing results (points cloud processing) [17]. The existing methods of geo-processing and analysis of images as well as high requirements to computation resources do not allow using the capabilities of technologies to the full extent [18]. Today the processing of laser scanning results (cloud of points) is frequently connected with equipment suppliers [19]. Though, the universal tools for processing and combining the clouds of points received from different sources are hardly available $[9,20]$.

The laser scanning clouds are presently collected and processed individually at the particular objects and territories according to process requirements (Xu et al., 2017). An automatic or semiautomatic segmentation and detection of objects in 3D laser scanning is the most resource-intensive step. There are also exist the special software packages developed for building particular types of models from the laser scanning data, such as Cyclone, RapidForm, 3Dipsos, all of them are intended for operation in 3D [4]. Besides, the suppliers of laser scanners offer to import the clouds of points to the most popular CAD systems, AutoCAD (Autodesk) and Microstation (Bentley). However, the processing of unprocessed data of 3D scanning in CAD environment is inconvenient, especially, if it is required to build 2D plans [21].

A complexity of historic buildings with an irregular geometry creates multiple problems in case of computer simulation and simulation of structural elements with different types of loads [22]. The people responsible shall take into account a number of possible and compatible solutions, which do not endanger the cultural significance of historic building [9]. Presently many publications offer to use the HBIM-based approach for solving the above problems. The first step of the modern HBIM is usually a remote acquisition of survey data with the use of laser scanner technologies in combination with digital photos [12, 22-24]. Therefore, there exist many examples of applications of laser scanning technologies for collecting data about historic buildings all over the world [9]. The scientific articles discuss the use of innovative laser scanning technologies for simulation and monitoring buildings of historic heritage, in order to forecast behavior thereof in the course of life cycle management. 


\section{Findings}

A unified hybrid technology for processing, storage, and visualization of laser scanning data and photo surveying of historic buildings is offered on the basis of algorithms of decoding and vectorization. The informational structure for HBIM models of historic heritage in SaintPetersburg (Russia) has been suggested. The first result of technology application demonstrated by the example of the historic building (water supply tower of Peter the Great St. Petersburg Polytechnic University) shows the robustness of the proposed technology. The developed technology can also be useful for processing the laser scanning data during engineering studies of roads and existing buildings.

\section{References}

1. H. Guan, J. Li, S. Cao, Y. Yu, Int. J. of Image and Data Fusion,7(3) (2016)

2. J. Heo, S. Jeong, H.-K. Park, J. Jung, S. Han, S. Hong, H.-G. Sohn, Computers, Environment and Urban Systems, 41 (2013)

3. C. Wang, Y.K. Cho, Automat. Constr., 49 (2015)

4. P. Tang, D. Huber, B. Akinci, R Lipman, A. Lytle, Automat. Constr., 19(7) (2010)

5. B. Yang, Z. Dong, ISPRS J Photogramm, 81 (2013)

6. O. Risbøl, C. Briese, M. Doneus, A. Nesbakken, J. Cult. Herit, 16(2) (2015)

7. M. Vaaja, J. Hyyppä, A. Kukko, H. Kaartinen, H. Hyyppä, P. Alho, Remote Sens-Basel, 3(3) (2011)

8. C. Cabo, C. Ordoñez, S. García-Cortés, J. Martínez, ISPRS J Photogramm, 87 (2014)

9. C. Dore, M. Murphy, International Archives of the Photogrammetry, Remote Sensing \& Spatial Information Sciences, 42(2W5), 185-192 (2017)

10. V. Badenko, D. Kurtener, V. Yakushev, A. Torbert, G. Badenko, Lect. Notes Comput. Sc, 9788, 57-69 (2016)

11. L. Barazzetti, Adv. Eng. Inform., 30(3) (2016)

12. R. Volk, J. Stengel, F. Schultmann, Automat. Constr., 38 (2014)

13. W. Mukupa, G.W. Roberts, C.M. Hancock, K. Al-Manasir, Surv. Rev., 49 (353) (2017)

14. A. Soni, S. Robson, B. Gleeson, Applied Geomatics, 7(2) (2015)

15. B. Quintana, S.A Prieto., A. Adán, A.S Vázquez, Adv. Eng. Inform., 30(4) (2016)

16. V.V. Lehtola, H. Kaartinen, A. Nüchter, R. Kaijaluoto, A. Kukko, P. Litkey, E. Honkavaara, T. Rosnell, M.T. Vaaja, J.-P. Virtanen, M. Kurkela, A. El Issaoui, L. Zhu, A. Jaakkola, J. Hyyppä, Remote Sensing, 9 (2017)

17. N. Hichri, C. Stefani, L. De Luca, P. Veron, G. Hamon, International Archives of the Photogrammetry, Remote Sensing \& Spatial Information Sciences, ISPRS Archives, 40(5W2) (2013)

18. A. Kukko, H. Kaartinen, J. Hyyppä, Y. Chen, Sensors, 12(9) (2012)

19. M. Lee, S. Lee, S. Kwon, S. Chin, KSCE J. Civ. Eng., 21(6) (2017)

20. X. Xiong, A. Adan, B. Akinci, D. Huber, Automat. Constr., 31 (2013)

21. L. Barazzetti, F. Banfi, R. Brumana, G. Gusmeroli, M. Previtali, G. Schiantarelli, Simul. Model Pract., 57, 71-87 (2015)

22. M. Murphy, E. Mcgovern, S. Pavia, Structural Survey, 27(4) (2009)

23. F. Chiabrando, M. Lo Turco, F. Rinaudo, International Archives of the Photogrammetry, 
Remote Sensing \& Spatial Information Sciences, ISPRS Archives, 42(2W5) (2017)

24. V. Badenko, D. Zotov, A. Fedotov, E3S Web of Conferences - EDP Sciences, 33, 01047 (2018) 